\title{
Claudicação em cavalos Crioulos atletas
}

\author{
Lameness in athletic Criollo horses
}

\section{Henrique Castagna de Abreu ${ }^{\mathrm{I}}$ Flávio Desessards De La Côrte ${ }^{\mathrm{II}}$ Karin Erica Brass $^{\mathrm{II}}$ Endrigo Pompermayer $^{\text {III }}$ Thiago Reis Ribeiro da Luz ${ }^{\text {III }}$ Diego De Gasperi ${ }^{\text {I }}$}

\section{RESUMO}

O objetivo deste trabalho foi identificar as principais causas de claudicação em cavalos Crioulos em treinamento para a prova do Freio de Ouro e competições de rédeas. Foram avaliados os registros clínicos de 201 cavalos Crioulos, 5,6 $\pm 2,1$ anos de idade, submetidos a exame físico devido à presença de claudicação ou histórico de baixo rendimento atlético entre os anos de 2002 a 2009. A claudicação foi localizada nos membros torácicos e pélvicos em 47,1\% $(n=105)$ e 52,9\% $(n=118)$, respectivamente. Nos machos, houve uma predominância de claudicação nos membros pélvicos $(60,0 \%)$ e nas fêmeas nos membros torácicos (57,1\%). Nos membros torácicos, 17,1\% (18/105) das alterações foram diagnosticadas proximais à articulação metacarpofalangeana, 14,3\% (15/105) na articulação metacarpofalangeana e 68,6\% (72/105) estavam localizadas distais à articulação metacarpofalangeana. Nos membros pélvicos, 78,8\% (93/118) apresentaram a origem da dor na região do tarso, 17,8\% (21/118) proximal ao tarso e 3,4\% (4) 118) distal ao tarso. As articulações interfalangeanas distais e intertarsiana distal/tarsometatarsiana são importantes fontes de dor e inflamação e estiveram mais frequentemente envolvidas em claudicações de membro torácico e pélvico, respectivamente. Os cavalos Crioulos apresentam problemas de claudicação semelhantes aos descritos em outras raças que participam de provas de rodeio.

Palavras-chave: claudicação, cavalos crioulos, sistema locomotor.

\section{ABSTRACT}

This study aimed to identify the source of lameness in Criollo horses that are competing in the most important discipline for this breed in Southern Brazil and also in reining competitions. Clinical records of adult Criollo horses $(n=201)$, $5.6 \pm 2.1$ years of age, that underwent lameness examination due to history of poor performance or lameness between 2002/ 2009 were analyzed. Lameness was diagnosed in the front limbs in $47.1 \%(n=105)$ and in the hind limbs $52.9 \%$ of the cases $(n=118)$. In males $60.0 \%$ of the lameness cases were located in the hind limbs. In females $57.1 \%$ of the lameness cases were located in the front limbs. In the front limbs $147.1 \%$, $n=105 / 201)$, problems proximal to the fetlock were diagnosed in $17.1 \%$ (18/105). Fetlock problems were diagnosed in $14.3 \%$ $(15 / 105)$ and lesions located distal to the fetlock level were seen in $68.6 \%$ (72/105) of the lameness cases diagnosed in the front limbs. In the hind limbs, problems located proximal to the tarsus were seen in $17.8 \%(21 / 118), 78.8 \%(93 / 118)$ in the tarsus and $3.4 \%$ (4/118) of the cases were seen distal to the tarsus. The distal interfalangeal joint in the front limbs and the distal tarsal joints in the hind limbs were the two most important sources of lameness. Criollo horses presented similar lameness problems as described in other show horses (reining, cutting and roping). This is the first lameness study done in Criollo horses and it will help to better understand the musculoskeletal diseases affecting this breed.

Key words: lameness, Criollo horses, locomotor system.

\section{INTRODUÇÃO}

Um estudo epidemiológico com 198 cavalos Puro Sangue de Corrida (PSC) demonstrou que 67,6\% dos casos de interrupção do treinamento estão relacionados a problemas de claudicação, seguidos dos

IMédico Veterinário Autonomo, Porto Alegre, RS, Brasil.

IIDepartamento de Clínica de Grandes Animais, Centro de Ciências Rurais (CCR), Universidade Federal de Santa Maria (UFSM), 97105-900, Santa Maria, RS, Brasil. E-mail: delacorte2005@yahoo.com.br. *Autor para correspondência.

IIIDoha Racing \& Equestrian Club, Doha, Qatar. 
problemas respiratórios (20,5\%) (ROSSDALE et al., 1985; HERNANDEZ \& HAWKINS, 2001).

As causas mais comuns de claudicação estão localizadas nos cascos, entretanto, a atividade a qual o animal é submetido tem uma grande influência no tipo e localização das lesões no sistema locomotor (MOYER \& CARTER, 2007). As alterações no terceiro metacarpiano (McIII), articulações do carpo e metacarpofalangeanas e ligamentos e tendões são as principais causas de descarte de cavalos de corrida e de pólo (SULLIVAN \& LUMSDEN, 2004; POSNER, 2004). Em cavalos de rodeio, apartação, tambor e rédeas, a síndrome do navicular, osteoartrite társica distal, desmite proximal do suspensório da articulação metacarpofalangeana (A-MTCF) e alterações relacionadas à articulação fêmurotíbiopatelar são as mais frequentes (DABAREINER et al., 2005a, 2005b; DYSON, 2007a; SCOTT, 2008). Ainda são escassos os dados que relacionam as afecções que causam claudicação com uma determinada disciplina.

Este trabalho teve o objetivo de identificar as principais causas de claudicação diagnosticadas em cavalos da raça Crioula em treinamento para competições de rédeas e provas do Freio de Ouro, que incluem provas de rédeas, apartação e manejo com o gado.

\section{MATERIAL E MÉTODOS}

Foram examinados 201 cavalos da raça

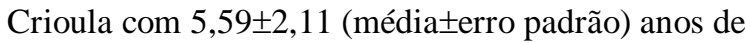
ambos os sexos $(62,33 \%$ machos - 5,52 $\pm 2,16$ anos; $37,67 \%$ fêmeas $-6,4 \pm 2,07$ anos de idade) provenientes de diversos centros de treinamento para provas do Freio de Ouro e rédeas. Os animais deste estudo apresentaram histórico de baixo rendimento atlético e/ ou claudicação e por esse motivo foram submetidos ao exame clínico do aparelho locomotor como previamente descrito (TURNER, 2007). O exame clínico foi realizado por um mesmo examinador e incluiu a inspeção, palpação, exame do cavalo em movimento, testes de flexão, bloqueios anestésicos e, quando necessário, exames complementares como radiografia, ultrassonografia e termografia. Os parâmetros analisados foram: idade, sexo, membro afetado (torácico/ pélvico), unilateral/bilateral (ou mais de dois membros afetados), grau de claudicação (AAEP, 1996), afecção encontrada. Foi realizada análise descritiva dos dados e teste qui-quadrado para identificar diferenças ao nível de $5 \%$.

\section{RESULTADOS E DISCUSSÃO}

Foram diagnosticadas 223 alterações do aparelho locomotor nos 201 cavalos que participaram deste estudo, uma vez que alguns cavalos possuíam afecções mistas, resultando no diagnóstico de mais de um achado clínico importante (Tabela 1). Afecções que resultaram em claudicação de membro torácico representaram 47,1\% (105/223) dos casos e de membro pélvico 52,9\% (118/223) (Figura 1A).

Com relação às fêmeas, $57,1 \%(48 / 84)$ apresentaram alterações no membro torácico, e 42,9\% (36/84) no membro pélvico. Já nos machos, 40,0\% (57/ 139) sofreram alterações no membro torácico, enquanto $60,0 \%$ (82/139) apresentaram problemas no membro pélvico $(\mathrm{P}<0,05)$ (Figura $1 \mathrm{~B})$. É possível que alguns aspectos relacionados ao manejo e treinamento sejam os fatores determinantes dessas diferenças entre os garanhões e as éguas da raça Crioula $\mathrm{O}$ excesso de peso nas éguas, situação comum quando encerram campanha nas exposições e ingressam nos treinamentos, poderia ser um fator importante, ao contrário dos garanhões, que geralmente iniciam os treinamentos após a temporada de reprodução nas manadas.

No momento do exame, $61.6 \%$ dos animais apresentavam uma claudicação de grau II (de uma escala de I a V) (AAEP, 1996), o que sugere um bom nível de percepção do problema por parte dos profissionais envolvidos, tanto para a claudicação nos membros torácicos como nos pélvicos. Quanto à apresentação das claudicações, 48,6\% eram unilaterais, $37,6 \%$ eram bilaterais e $13,8 \%$ envolviam mais de dois membros. Essa distribuição foi diferente entre machos (mais casos unilaterais) e fêmeas (distribuição uniforme $)(\mathrm{P}<0,05)$.

Das alterações identificadas nos membros torácicos (Figura 1C), apenas 17,1\% (18/105) estavam localizadas proximal à articulação metacarpofalangeana, mostrando que lesões proximais, como na articulação cárpica, são menos frequentes no cavalo Crioulo do que nos cavalos de corrida, por exemplo (ROSS, 2003). Diferente dos cavalos PSC, os problemas de carpo foram pouco frequentes ( $2 \%$ ) nos animais deste estudo, assim como a ocorrência de problemas nas articulações rádiocarpiana e intercarpiana em cavalos que não participam de corridas nãoé comum (DYSON, 2007a).

A ocorrência de problemas nos metacarpianos (Mc) acessórios nos cavalos deste estudo foi limitada à periostite do segundo metacarpiano em 8/105 casos (McII) $(7,6 \%)$, sendo bem inferior aos problemas que acometem os cavalos PSC, nos quais a doença metacarpiana dorsal (McIII) é a 
Tabela 1 - Frequência de afecções do sistema locomotor diagnosticadas nos membros torácicos (n=105) e pélvicos (n=118) de 201 cavalos Crioulos em treinamento.

\begin{tabular}{|c|c|c|c|c|}
\hline \multirow[b]{2}{*}{ Afecções } & \multicolumn{2}{|c|}{ Membro torácico } & \multicolumn{2}{|c|}{ Membro pélvico } \\
\hline & $\mathrm{n}$ & $\%$ & $\mathrm{n}$ & $\%$ \\
\hline Abscesso subsolar casco & 4 & 3,8 & & \\
\hline Artrite articulação interfalangeana distal & 2 & 1,9 & & \\
\hline Artrite/trauma carpo & 2 & 1,9 & & \\
\hline Calcificação cartilagem complementares falange distal & 1 & 1,0 & & \\
\hline Cascos doloridos (sem diagnostico específico) & 10 & 9,5 & & \\
\hline DAD/sinovite escápulo-umeral & 2 & 1,9 & & \\
\hline DAD/sinovite tíbiotársica & & & 1 & 0,9 \\
\hline Desmite proximal/ramo do lig. suspensório do boleto articulação metacarpofalangeana & 4 & 3,8 & & \\
\hline Exostose dorso proximal falange proximal & 3 & 2,9 & & \\
\hline Fratura falange distal & 2 & 1,9 & & \\
\hline Laminite crônica (com rotação da falange distal) & 3 & 2,9 & & \\
\hline Miopatia/mialgia região glutea & & & 11 & 9,3 \\
\hline Osteíte falange distal & 7 & 6,7 & & \\
\hline Periostite proliferativa McII/McIII/MtIV & 8 & 7,6 & 1 & 0,9 \\
\hline Rabdomiólise por esforço & & & 2 & 1,7 \\
\hline Rachadura profunda do casco & 1 & 1,0 & & \\
\hline Ruptura Peroneus tersius & & & 1 & 0,9 \\
\hline Sesamoidite & 1 & 1,0 & & \\
\hline Síndrome do navicular (com evidência radiográfica) & 3 & 1,3 & & \\
\hline Sinovite art. interfalangeana proximal & 1 & 1,0 & & \\
\hline Sinovite art. interfalangeana distal & 35 & 15,7 & & \\
\hline Sinovite art. tíbio társica & & & 1 & 0,9 \\
\hline Sinovite/artrite art. fêmurotíbiopatelar & & & 7 & 5,9 \\
\hline Sinovite/artrite boleto articulação metacarpofalangeana & 7 & 6,7 & & \\
\hline Tarsite/osteoartrite distal & & & 91 & 77,1 \\
\hline Tendinite tendão flexor digital profundo & 3 & 2,9 & & \\
\hline Tenossinovite bainha digital flexora & 5 & 4,8 & 3 & 2,5 \\
\hline Tenossinovite séptica bainha digital flexora & 1 & 1,0 & & \\
\hline Total & 105 & $100 \%$ & 118 & $100 \%$ \\
\hline
\end{tabular}

DAD: doença articular degenerativa.

mais importante em potros iniciando o treinamento (2 anos de idade) (NUNAMAKER \& PROVOST, 1991) e pode variar entre $70 \%$ e $80 \%$ (STOEVER, 1992). A maioria das reações proliferativas do McII (secundárias à hemorragia subperiosteal e descolamento do periósteo) em animais em treinamento é decorrente de traumas infligidos pelo membro contralateral (interferência) (DYSON, 2007b).

Alterações relacionadas à articulação metacarpofalangeana no membro torácico apresentaram uma frequencia de 14,3\% (15/105) (Figura 1C), semelhantes aos observados em potros PSC (ROSSDALE et al., 1985). SMITH(1997) e NOBLE(2001) concordam que as alterações nessa articulação não são as lesões mais comuns em cavalos de rodeio e apartação.
Neste estudo, alterações distais à articulação metacarpofalangeana no membro torácico representaram 68,6\% (72/105) dos casos, com a grande maioria sendo relacionada com os cascos dos membros torácicos (Figura 1C). Destas, 48,6\% (35/72), ou seja, quase a metade foram casos diagnosticados como sinovite da articulação interfalangeana distal (AID). McILWRAITH (2002) define a sinovite como sendo a inflamação da membrana sinovial de uma articulação. Defeitos de conformação associados ao trauma repetitivo gerado pelo treinamento são fatores desencadeantes dessa alteração, que também pode estar relacionada com alterações da região podotroclear, geralmente associada à tendinite do flexor digital profundo (próximo à inserção na falange distal), desmite do ligamento ímpar ou dos colaterais do osso navicular, osteíte e doença do navicular (TURNER, 2007). A 


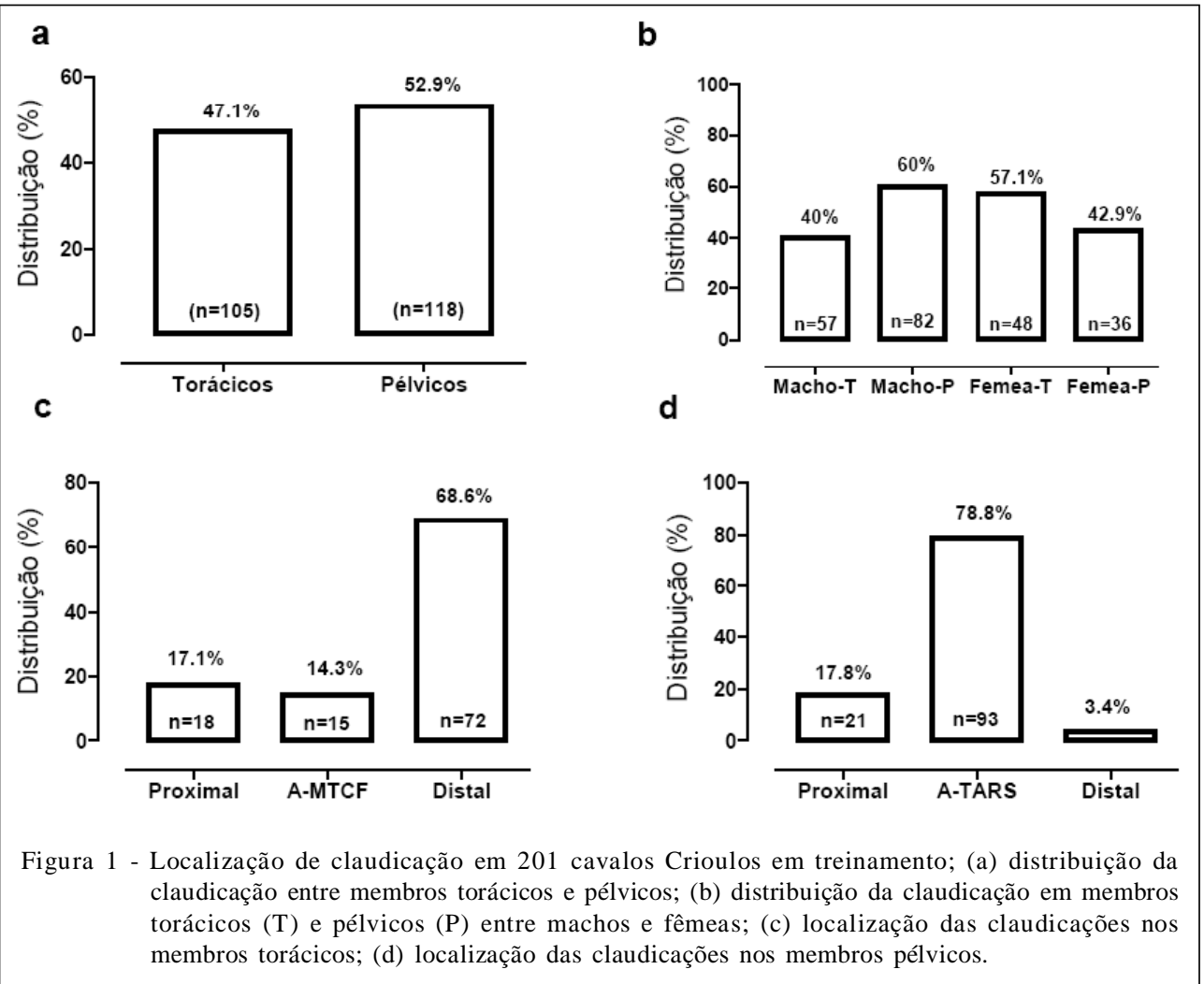

frequencia de casos confirmados pelo exame clínico e radiografia envolvendo o aparelho podotroclear $(2,9 \%)$ foi considerada muito baixa. Entretanto, não se deve ignorar a possibilidade de que alguns casos de sinovite da interfalangeana distal neste estudo estejam também relacionados com doença do navicular e doença articular degenerativa da articulação interfalangeana distal, apenas não investigados mais detalhadamente (SCOTT, 2008).

Problemas nos cascos não são exclusivos de cavalos PSC, nos quais aproximadamente $20 \%$ das causas de claudicação localizam-se nos cascos (ROSSDALE et al., 1985). Das claudicações de membro torácico nos Crioulos, 30,5\% (32/105) estavam relacionados com os cascos. A alta frequência observada de problemas nessa região não é surpresa se considerarmos que $87 \%$ dos cavalos Crioulos em treinamento documentados em outro estudo apresentavam talões contraídos, o que diminui a elasticidade e dificulta a expansão normal deste durante a fase de apoio, predispondo a lesões (DO CANTO et al., 2006). Problemas de conformação dos cascos foram observados em 80,9\% (85/105) das claudicações de membro torácico nos cavalos Crioulos deste estudo, sem levar em consideração o tipo e localização da alteração principal que causou a claudicação. Defeitos de conformação dos cascos também foram documentados na raça Quarto-de-Milha, nos quais 78\% estavam associados com ângulos diferentes nos cascos (DABAREINER et al., 2005b). Os problemas de conformação aqui identificados foram: eixo quebrado para trás $(15,23 \%$; 16/105), cascos longos $(26,66 \% ; 28 /$ $105)$, talões contraídos $(5,71 \% ; 6 / 105)$ e talões escorridos $(1,9 \% ; 2 / 105)$. Além dos $87 \%$ dos animais apresentarem talões contraídos, DO CANTO et al. (2006) ainda descreveram $49 \%$ com desequilíbrio médio lateral, $11 \%$ com eixo quebrado para trás e $23 \%$ com ângulo dos cascos contralaterais diferentes.

Diferentemente dos achados em cavalos de rodeio (DABAREINER et al., 2005a; 2005b), nos cavalos Crioulos deste estudo, as claudicações ocorreram mais frequentemente nos membros pélvicos do que nos torácicos. Talvez a concentração de exercícios sobre os membros pélvicos, característica da modalidade na qual competem, predisponha os cavalos Crioulos a mais problemas nestes do que nos torácicos. Com relação às alterações nos membros pélvicos (Figura 1D), as localizadas proximais ao tarso representaram 17,8\% (21/ 118), sendo em sua maioria diagnosticadas como sinovite ou osteoartrite da articulação fêmurotíbiopatelar $(33,3 \%$; $7 / 21)$ e miopatia dos grandes grupos musculares da região glútea $(38,1 \% ; 8 / 21)$. Essas 
lesões também são bastante descritas em cavalos que participam de competições de rodeio nos Estados Unidos. Nestes, as principais causas de mialgias e miopatias da região glútea são atribuídas a fatores como: inflamação das articulações do tarso ou fêmurotibiopatelar, excesso de peso ou inexperiência do cavaleiro, e ajuste incorreto dos equipamentos de montaria (NOBLE, 2001). Portanto, recomenda-se que as causas primárias sejam identificadas para que o tratamento das mialgias secundárias seja eficiente.

A região da articulação do tarso foi o local da origem das claudicações em 78,8\% (93/118) dos casos, sendo que a inflamação das articulações intertarsiana distal e tarsometatarsiana (tarsite distal) foi a afecção mais frequentemente diagnosticada nos cavalos Crioulos. Em cavalos de outras raças que competem em rodeio, apartação, laço e corrida de tambores, as inflamações e osteoartrites das articulações distais do tarso também são extremamente comuns (GALLEY, 2001; JACKMAN, 2001; NOBLE, 2001; DABAREINER et al., 2005a, 2005b; SCOTT 2008). Isso ocorre porque grande parte do peso do animal é transferida para os membros pélvicos durante a execução de manobras que envolvem rápida mudança de direção e paradas bruscas, colocando stress e torque nas articulações do tarso. O diagnóstico dessas alterações é primariamente clínico, mesmo em animais com pouca ou nenhuma alteração radiológica evidente, pois não existe uma boa correlação entre os achados radiológicos e o grau de claudicação (NOBLE, 2001; BYAM-COOK \& SINGER, 2009). CURRIE (1997) analisou os principais fatores de baixo rendimento em esbarradas nos cavalos de rédeas e constatou que, quando a causa era claudicação, a origem da dor era predominantemente na região do tarso, mostrando a semelhança das lesões encontradas em cavalos Crioulos e de rédeas nos Estados Unidos, onde predomina a raça Quarto de Milha.

Lesões distais ao do tarso representaram apenas $3,4 \%$ (4/118), mostrando claramente que problemas distais ao tarso não foram frequentes nos cavalos Crioulos deste estudo, bem como nos relatos envolvendo cavalos de rédeas, apartação, corrida de tambores e laço que fazem parte das provas de rodeio nos Estados Unidos.

Esta é a primeira descrição das principais afecções do sistema músculo-esquelético que afetam cavalos Crioulos em competições. Os achados clínicos demonstraram que as lesões nos cavalos Crioulos são muito semelhantes às descritas em cavalos que participam de provas semelhantes nos Estados Unidos (CURRIE, 1997; DABAREINER et al., 2005a, 2005b; SCOTT, 2008). Sabe-se que, em cavalos de rédea, a individualidade no treinamento também determina o tipo de lesão encontrada nos cavalos entre diferentes treinadores (ANDERSON, 1997). Entretanto, mais estudos serão necessários para avaliar o papel do método/treinador na ocorrência e distribuição das causas de claudicações em cavalos Crioulos.

\section{CONCLUSÃO}

Os Cavalos crioulos apresentam as mesmas afecções citadas em outros estudos envolvendo cavalos de rodeio nos Estados Unidos (western performance horses), provavelmente porque realizam manobras e competem em modalidades muito semelhantes.

\section{REFERÊNCIAS}

AAEP. Guide for veterinary service and judging of equestrian events. 5ed. Lexington, KY: American Association of Equine Practitioners, 1996. 63p.

ANDERSON, A. Reining. In: ANNUAL CONVENTION OF THE AMERICAN ASSOCIATION OF EQUINE PRACTITIONERS, 43., 1997, Phoenix. Proceedings... Lexington: AAEP, 1997. p.35-36. Disponível em: <http:// www.ivis.org/proceedings/AAEP/1997/Anderson.pdf>. Online. Acesso em: 27 dez. 2010.

BYAM-COOK, K.L.; SINGER, E.R. Is there a relationship between clinical presentation, diagnostic and radiographic findings in horses with osteoarthritis of the small tarsal joints? Equine Veterinary Journal, v.41, n.2, p.118-123, 2009. Disponível em: <http://onlinelibrary.wiley.com/doi/10.2746/ 042516408X345107>. Acesso em: 23 de dez. 2010. doi: $10.2746 / 042516408 \times 345107$.

CURRIE, A.K. The western performance horse. In: ANNUAL CONVENTION OF THE AMERICAN ASSOCIATION OF EQUINE PRACTITIONERS, 43., 1997, Phoenix. Proceedings... Lexington: AAEP, 1997. p.46-48. Disponível em: 〈http://www.ivis.org/proceedings/AAEP/1997/Currie.pdf>. Online. Acesso em: 27 dez 2010.

DABAREINER, R.M. et al. Lameness and poor performance in horses used for team roping: 118 cases (2000-2003). Journal of the American Veterinary Medical Association, v.226, n.10, p.1694-1699, 2005a.

DABAREINER, R.M. et al. Musculoskeletal problems associated with lameness and poor performance in horses used for barrel racing: 118 cases (2000-2003). Journal of the American Veterinary Medical Association, v.227, n.10, p.1646-1650, 2005b.

CANTO, L.S. et al. Frequência de problemas de equilíbrio nos cascos de cavalos crioulos em treinamento. Brazilian Journal Veterinary Research Animal Science, v.43, n.4, p.489495, 2006. Disponível em: <http://www.revistasusp.sibi.usp.br/ scielo.php? script=sci_arttext\&pid=S1413 95962006000400008\&lng=pt\&nrm=iso>. Acesso em: 25 dez. 2010. 
DYSON, S.J. Some carpal lesions in the non-racehorse. In: ANNUAL CONVENTION OF THE AMERICAN ASSOCIATION OF EQUINE PRACTITIONERS, 53., 2007a, Orlando. Proceedings... Lexington: AAEP, 2007a. p.99103. Disponível em: <http://www.ivis.org/proceedings/ aaepfocus/2007/dyson2.pdf>. Online. Acesso em: 27 dez. 2010.

DYSON, S.J. Diagnosis and management of common suspensory lesions in forelimbs and hindlimbs of sport horses. Clinical techniques in equine practice, v.6, n.3, p.179-188, 2007b. Disponível em: < h t p : / / w w w. s c i e n c e d i r e c t. c o m/ science?_ob=MImg\&_imagekey=B 7 GW6-4PYN33H-6$1 \&$ \&_di $=20450 \&$ \& user $=687358 \&$ \& pii $=$ S 153475160 7000339\&_origin $=$ browse \&_zone $=$ rslt_list_item\&_coverD ate $=09 \% 2 \mathrm{~F} 30 \% 2 \mathrm{~F} 2007 \& \_s k=999939996 \& \mathrm{wchp}=\mathrm{dGLzVtz}-$ zSkWb\&md5=f4b353d93b74d5570680853dc9bb8f44\&ie=/sdarticle> Acesso em: 23 dez. 2010. doi: 10.1053/j.ctep.2007.08.004.

GALLEY, R.H. Injuries of the team roping horse. In: ANNUAL CONVENTION OF THE AMERICAN ASSOCIATION OF EQUINE PRACTITIONERS, 47., 2001, San Diego. Proceedings... Lexington: AAEP, 2001. p.15-21. Disponível em: <http:// www.ivis.org/proceedings/AAEP/2001/91010100015.pdf>. Online. Acesso em: 27 dez. 2010

HERNANDEZ, J.; HAWKINS, D.L. Training failure among yearling horses. American Journal of Veterinary Research, v.62, n.9, p.1418-1422, set.2001. Disponível em: <http:// avmajournals.avma.org/doi/pdf/10.2460/ajvr.2001.62.1418>. Acesso em: 23 dez. 2010. doi: 10.2460/ajvr.2001.62.1418.

JACKMAN, B.R. Common lameness in the cutting and reining horse. In: ANNUAL CONVENTION OF THE AMERICAN ASSOCIATION OF EQUINE PRACTITIONERS, 47., 2001, San Diego. Proceedings... Lexington: AAEP, 2001. p.6-11. Disponível em: <http://www.ivis.org/proceedings/AAEP/2001/ 91010100006.pdf>. Online. Acesso em: 27 dez. 2010.

McILWRAITH, C.W. Diseases of joints, tendons, ligaments and related structures. In: STASHAK, T.S. Adams lameness in horses. 5.ed. Philadelphia: Lippincott Willians \& Wilkins, 2002. Cap.7, p.417-601.

MOYER, W.A; CARTER, G.K. Diagnostic evaluation of equine foot. In: FLOYD, A.E; MANSMANN, R.A. Equine podiatry. Saint Louis: Saunders, 2007. Cap.8, p.112-127.

NOBLE, J.K. Lameness in the western pleasure horse. In: ANNUAL CONVENTION OF THE AMERICAN ASSOCIATION OF EQUINE PRACTITIONERS, 47., 2001, San Diego. Proceedings... Lexington: AAEP, 2001. p.1214. Disponível em: <http://www.ivis.org/proceedings/AAEP/ 2001/91010100012.pdf>. Online. Acesso em: 27 dez. 2010.
NUNAMAKER, D.M; PROVOST, M.T. The bucked shins complex revised. In: ANNUAL CONVENTION OF THE AMERICAN ASSOCIATION OF EQUINE PRACTITIONERS, 37., 1991, San Francisco. Proceedings... Lexington: AAEP, 1991. V.37. 996p. p.757-762

POSNER, R.E. Veterinary aspects of training and competing polo ponies. In: HINCHCLIFF, K.W. et al. Equine sports medicine and surgery. Saint Louis: Saunders, 2004. Cap.53, p.1118-1122.

ROSS, M.W. Carpus. In: DYSON, S.J; ROSS, M.W. Diagnosis and management of lameness in the horse. Saint Louis: Saunders, 2003. Cap.39, p.376-394.

ROSSDALE, P.D. et al. Epidemiological study of wastage among racehorses: 1982-1983. Veterinary Record, v.116, n.3, p.6669, 1985. Disponível em: <http://veterinaryrecord.bmj.com/ content/116/3/66.abstract>. Acesso em: 23 dez 2010. doi:10.1136/vr.116.3.66.

SCOTT, M. Musculoskeletal injuries in nonracing Quarter Horses. Veterinary Clinics of North America: Equine Practice, v.24, p.133-152, 2008. Disponível em: <http://www.sciencedirect.com/ science?_ob=ArticleURL\&_udi=B7RM 8-4RY 8767 C\&_user $=687358 \&$ \& coverDate $=04 \% 2$ F3 $0 \% 2$ F $2008 \&$ _rdoc $=1 \& \_$fmt=high\&_orig=search\&_origin=search\&_sort=d\&_doc anchor $=\&$ view $=$ c\&_searchStrId $=1587543369 \& \_$rerunOrigin $=$google \&_acct=C000037899\&_version=1\&_urlVersion=0\&_userid $=687$ $358 \& \mathrm{md} 5=8229 \mathrm{f} 2 \mathrm{~b} 30 \mathrm{f} 7671 \mathrm{be} 68 \mathrm{bb} 09 \mathrm{ee} 74 \mathrm{cced} 32 \&$ searchtype $=a>$. Acesso em: 23 dez. 2010. doi:10.1016/j.cveq.2007.11.006.

SMITH, H.A. Cutting. In: ANNUAL CONVENTION OF THE AMERICAN ASSOCIATION OF EQUINE PRACTITIONERS, 43., 1997, Phoenix. Proceedings... Lexington: AAEP, 1997. p.31-34. Disponível em: <http://www.ivis.org/proceedings/ AAEP/1997/Smith.pdf>. Online. Acesso em: 27 dez. 2010.

STOEVER, S.M. Contribution of microfractures to dorsal metacarpal disease. In: ANNUAL CONVENTION OF THE AMERICAN ASSOCIATION OF EQUINE PRACTITIONERS, 38., 1992, Lexington. Proceedings... Lexington: AAEP, 1992. v.38. 763p. p.3.

SULLIVAN, C.B; LUMSDEN, J.M. Veterinary aspects of racing and training thoroughbred race horses. In: HINCHCLIFF, K.W. et al. Equine sports medicine and surgery. Saint Louis: Saunders, 2004. Cap.49, p.1051-1072.

TURNER, T.A. Caudal hoof lameness. In: FLOYD, A.E; MANSMANN, R.A. Equine podiatry. Saint Louis: Saunders, 2007. Cap.14, p.294-312. 